\title{
Acalculous Perforated Hemorrhagic Emphysematous Cholecystitis Caused by Clostridium perfringens in a Patient with Myeloma Taking Daratumumab
}

\author{
Allison E. Berndtson, Michelle G. Hamel, Todd W. Costantini, and Raul Coimbra
}

\begin{abstract}
Background: Acute calculous cholecystitis is a common disease entity familiar to every surgeon, however, variations such as acalculous cholecystitis, hemorrhagic cholecystitis, and emphysematous or gangrenous cholecystitis are rare. Diagnosis is more difficult and delays increase patient morbidity.

Case Presentation: We present the case of a 75-year-old female Caucasian with multiple myeloma, undergoing treatment with daratumumab and dexamethasone, who was diagnosed with acute acalculous, emphysematous, perforated, and hemorrhagic cholecystitis. Active hemorrhage was demonstrated via contrast blush on computed tomography (CT) scan. She was treated expeditiously and recovered well. Cultures demonstrated a polymicrobial infection, including Clostridium perfringens.

Conclusion: This is a complex case with unclear initial etiology, likely exacerbated by growth of Clostridium perfringens. It is possible that the patient's immunocompromised state contributed to infection that then lead to gallbladder emphysema, perforation, and hemorrhage. Thus, the clinician should be aware of the possibility of complicated cholecystitis in the immunocompromised patient, even in the absence of neutropenia, to avoid missing devastating complications of perforation or hemorrhage.
\end{abstract}

Keywords: hemorrhagic cholecystitis; emphysematous cholecystitis; acalculous cholecystitis; myeloma; daratumumab

\begin{abstract}
A CUTE CALCULOUS CHOLECYSTITIS is the most common complication of cholelithiasis, accounting for thousands of cholecystectomies per year in the United States. Many causative organisms have been identified, most frequently gram-negative bacilli, streptococci, and anaerobes [1]. Subtypes of cholecystitis exist in different patient populations. Acalculous cholecystitis, or cholecystitis in the absence of gallstones, is a rare illness that occurs most commonly in critically ill patients receiving total parenteral nutrition (TPN) when the gallbladder is not routinely stimulated to contract. Anaerobes including Enterobacteriaceae are the most frequently cultured organisms in this population. Hemorrhagic cholecystitis is even less common, most often presenting in patients with coagulopathy or trauma [2] al-
\end{abstract}

though other etiologies such as infection and malignancy have been described [3]. Presentation often includes hemobilia or hematemesis, although this may not be present if a clot obstructs the cystic duct. Diagnosis is difficult and timesensitive intervention critical. Finally, severe infection can lead to gallbladder wall emphysema, necrosis, gangrene, and perforation. These patients are often severely ill with an increased risk of mortality, and treatment is considered a surgical emergency.

\section{Case Presentation}

We report the case of a 75-year-old Caucasian female undergoing treatment for multiple myeloma, who presented 
with $12 \mathrm{~h}$ of acute epigastric pain, nausea, and vomiting. She had felt well the previous day until consuming a high-fat meal, then developed constant, worsening epigastric pain. This was associated with bilious, non-bloody emesis and several episodes of non-bloody diarrhea. She was brought to the emergency department by her family early the next morning for persistent pain, chills, diaphoresis, and altered mental status. Upon further questioning the family noted that the patient had complained of episodic, crampy epigastric pain several times over the last month, each episode lasting three to four minutes and self-limited. These were often but not always associated with fatty food. To their knowledge the patient did not have a history of jaundice, icterus, acholic stools, or dark urine.

Other than the active myeloma, the patient had a known, asymptomatic meningioma, and a recent episode of shingles that had resolved. She had undergone no prior abdominal surgery. Family and social history were unremarkable. Her myeloma treatment consisted of weekly daratumumab, an anti-CD38 monoclonal antibody, and dexamethasone though she reported that her last dose of each was $10 \mathrm{~d}$ prior. She denied use of other medications.

Upon evaluation in the emergency department the patient was found to be afebrile, hypertensive at $151 / 101 \mathrm{~mm} \mathrm{Hg}$ with a normal heart rate of 96, and mildly tachypnic at a rate of 24. She appeared to be in mild distress, was diaphoretic, and was oriented only to self and place. Her abdomen was soft and non-distended, but moderately tender to deep palpation in the epigastric region. Her gallbladder was not palpable. She had no peritoneal signs.

Prior to consultation of the surgical team, the patient had laboratory studies and imaging performed in the emergency department. Laboratory studies were notable for a normal white blood cell count at 7.4, normal liver function tests and lipase, and an elevated lactate of $4.7 \mathrm{mmol} / \mathrm{L}$. Her blood glucose was elevated at $184 \mathrm{mg} / \mathrm{dL}$ without a history of dia- betes. She had no coagulopathy or thrombocytopenia. Imaging consisted of a chest radiograph, non-contrast computed tomography (CT) of the head and CT of the abdomen-pelvis with intravenous (IV) contrast that were completed prior to surgical consultation. The chest radiograph was unremarkable, whereas the head CT demonstrated only her known meningioma. The CT scan of her abdomen was immediately reviewed by the surgical team and demonstrated typical signs of acute cholecystitis, including a markedly thickened gallbladder wall. In addition, a single small focus of gas was noted within the gallbladder wall, with an associated contrast blush that layered within the gallbladder lumen (Fig. 1). Together these findings were indicative of either emphysematous cholecystitis with active hemorrhage or an emphysematous hemocholecyst.

The patient was immediately taken for operative intervention after informed consent was obtained from her family. She underwent an open cholecystectomy given her active hemorrhage as well as the size and distension of her gallbladder. Intra-operative findings confirmed acute hemorrhagic cholecystitis. Free bloody-bilious fluid was also noted within the abdominal cavity consistent with gallbladder perforation. Cholecystectomy proceeded without evident complication. After removal, the gallbladder was opened and no stones were identified. Bile cultures were sent. The patient tolerated the procedure well without significant hypotension or blood loss. A drain was left in the gallbladder fossa.

The patient recovered well from her operation. She remained hemodynamically stable and had rapid return to her baseline normal mental status. She was able to tolerate a regular diet within three days. Her drain was removed on postoperative day two after output became minimal and remained non-bilious. She was discharged on post-operative day five.

Final pathology confirmed acute hemorrhagic cholecystitis with ulceration, abscess formation, and tissue necrosis (Fig. 2). Serial sectioning revealed hemorrhage primarily at the

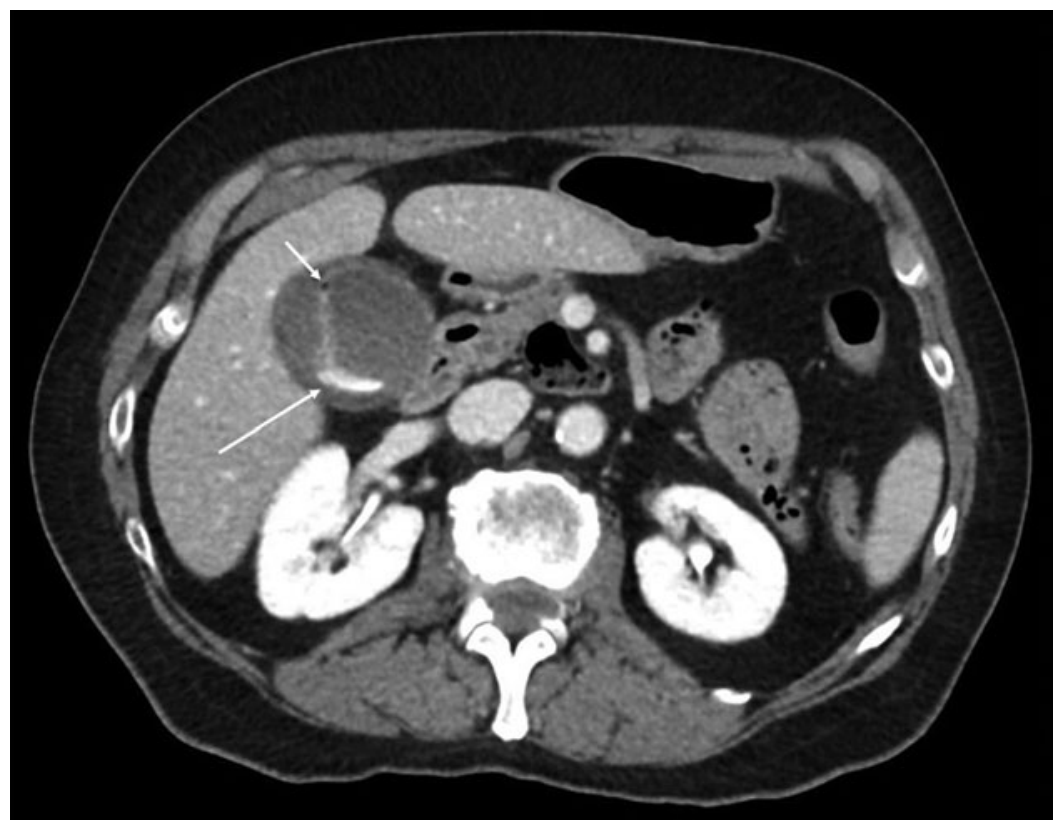

FIG. 1. Computed tomography (CT) abdomen-pelvis with intravenous (IV) contrast. Small arrow demonstrates focus of air within the gallbladder wall. Large arrow indicates pooling IV contrast within the gallbladder lumen. 


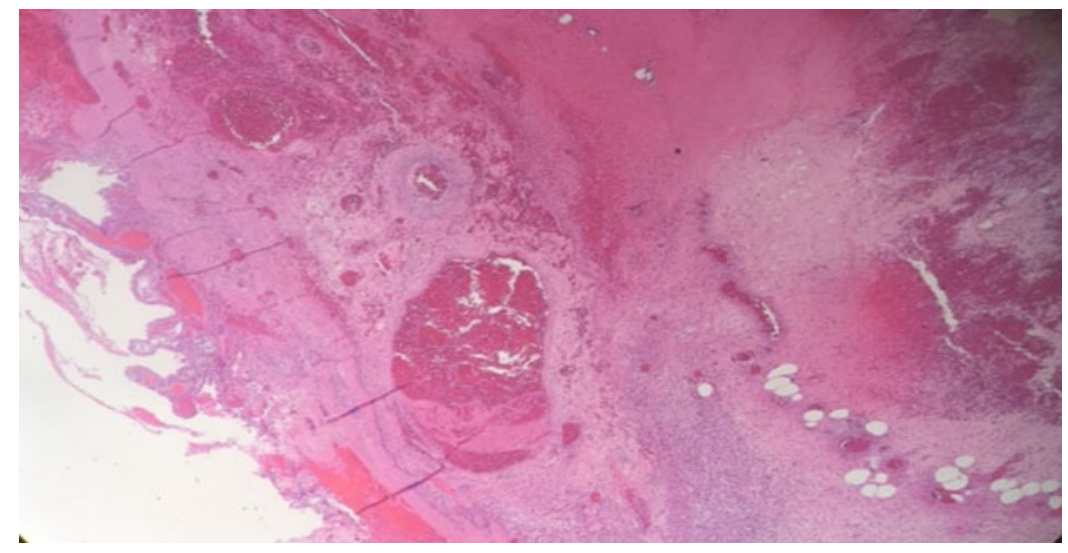

FIG. 2. Microscopic pathology of the gallbladder wall demonstrating hemorrhage and ulceration.

gallbladder neck, with multiple cystic spaces filled with coagulated blood. Vasculitis was noted in the areas of abscess formation. No stones were identified, but the cystic duct was occluded at the gallbladder neck by thrombus. Intra-operative bile swabs grew Citrobacter species, Enterobacter cloacae, and Enterococcus faecium on aerobic culture and Clostridium perfringens on anaerobic culture. Her antibiotic regimen consisted of piperacillin-tazobactam, which was started pre-operatively in the emergency department and continued peri-operatively for $24 \mathrm{~h}$ given the suspicion for gallbladder perforation. Antibiotics were discontinued subsequently because the infected organ had been removed. She did not develop signs of any subsequent infection.

\section{Discussion and Literature Review}

This patient had a complex diagnosis with potential for significant morbidity, yet recovered quickly and without complication. Her recovery was certainly aided by rapid diagnosis and treatment. Although ultrasound is typically the diagnostic modality of choice for cholecystitis, the CT scan obtained proved useful in this patient because the visible contrast blush and focus of air immediately showed the hemorrhagic and emphysematous nature of her disease process. This led to rapid activation of the operating room without the delay that might otherwise have been present had the severity of her condition been unrecognized.

We find the most interesting aspect of this case to be the unclear etiology of her disease. Her gallbladder contained no stones, making this a diagnosis of acute acalculous cholecystitis; however, our patient did not demonstrate any of the usual risk factors such as TPN use, prolonged nothing by mouth state, or critical illness. Whereas acalculous cholecystitis has been described in patients with immunodeficiency caused by human immunodeficiency virus (HIV), there are few case reports in patients immunosuppressed as a result of other causes. Kassar et al. [4] and Pielacinski et al. [5] describe acalculous cholecystitis arising during chemotherapy for acute myeloblastic and lymphoblastic leukemia, respectively; both patients were neutropenic at the time of diagnosis. Interestingly, there are also reports of acalculous cholecystitis without associated neutropenia in patients receiving small molecule inhibitors for solid organ tumors, such as sunitinib and sorafenib [6,7]. We found only a single case report of acalculous cholecystitis in a patient on monoclonal antibody therapy, that of a 15-year-old female on infliximab for juvenile polyarticular rheumatoid arthritis. Overall daratumumab has relatively few infectious complications reported thus far, primarily pulmonary [8], with only a single article reporting a gastrointestinal tract infection [9]. Our patient was additionally on dexamethasone but with only intermittent dosing, the last administration of which was $10 \mathrm{~d}$ prior to presentation.

Although our patient did not have gallstones that could have obstructed the cystic duct, pathologic findings did lead to the possibility of cystic duct obstruction caused by clots. This raises the question of which came first: infection leading to hemorrhage, or hemorrhage occluding the duct and infection developing in an obstructed gallbladder? Despite the occlusive thrombus we believe that the active contrast blush seen on CT argues for initial infection leading to hemorrhage within the gallbladder as the bleeding visualized on the CT scan clearly emanates from the single focus of emphysema.

Vasculitis or vascular occlusion have also been raised as possible etiologies of acute emphysematous cholecystitis. May and Strong [10] considered the possibility of endarteritis obliterans or cystic artery occlusion in their 1971 article, whereas Hernandez-Rodriguez et al. discussed single-organ vasculitis [11]. The microscopic pathology on our patient did demonstrate vasculitis present within areas of abscess formation; unfortunately which developed first cannot be definitively determined. Interestingly, May and Strong [10] did note that Escherichia coli and C. perfringens were the organisms cultured most commonly from emphysematous cholecystitis in their study, which is consistent with microbiologic studies from our patient.

Finally, once infection had begun we believe that the presence of $C$. perfringens likely contributed to both the emphysematous and hemorrhagic nature of her disease process. Clostridium perfringens is well known for its invasive, necrotizing, and gas-forming properties as the etiologic agent of peripheral gas gangrene. Once invaded into the gallbladder wall it is expected that hemorrhage could arise as cystic artery branches are damaged. This is consistent with our image of hemorrhage arising directly from the single focus of emphysema.

\section{Conclusion}

This case report describes a 75-year-old female with multiple myeloma receiving monoclonal antibody therapy who 
developed acalculous perforated hemorrhagic emphysematous cholecystitis of unclear etiology. It is possible that the patient's immunocompromised state contributed to infection with $C$. perfringens leading to gallbladder emphysema, perforation, and hemorrhage. Thus, the clinician should be aware of the possibility of complicated cholecystitis in the immunocompromised patient, even in the absence of neutropenia, to avoid missing devastating complications of perforation or hemorrhage.

\section{Author Disclosure Statement}

All authors state that they have no disclosures, and that no competing financial interests exist.

\section{References}

1. Claesson BE, Holmlund DE, Matzsch TW. Microflora of the gallbladder related to duration of acute cholecystitis. Surg Gynecol Obstet 1986;162:531-535.

2. Parekh J, Corvera CU. Hemorrhagic cholecystitis. Arch Surg 2010;145:202-204.

3. Ku J, DeLaRosa J, Kang J, et al. Acute cholecystitis with a hemocholecyst as an unusual presentation of gallbladder cancer: report of a case. Surg Today 2004; 34:973-976.

4. Kassar O, Kallel F, Ghorbel M, et al. Acute acalculous cholecystitis complicating chemotherapy for acute myeloblastic leukemia. Leuk Res Rep 2015;4:39-41.

5. Pielacinski K, Ejduk A, Wroblewski T, et al. Laparoscopic cholecystectomy for acalculous cholecystitis in a neutropenic patient after chemotherapy for acute lymphoblastic leukemia. Wideochir Inne Tech Maloinwazyjne 2014;9: 468-472.

6. da Fonseca LG, Barroso-Sousa R, Sabbaga J, et al. Acute acalculous cholecystitis in a patient with metastatic renal cell carcinoma treated with sunitinib. Clin Pract 2014; 4:635.

7. Aihara Y, Yoshiji H, Yamazaki M, et al. A case of severe acalculous cholecystitis associated with sorafenib treatment for advanced hepatocellular carcinoma. World J Gastrointest Oncol 2012;4:115-118.

8. Oliva S, Palumbo A. Monoclonal antibodies for treating multiple myeloma-A new era, new safety considerations? Expert Opin Drug Safe 2016;15:1-6.

9. Plesner T, Arkenau HT, Gimsing P, et al. Phase 1/2 study of daratumumab, lenalidomide, and dexamethasone for relapsed multiple myeloma. Blood 2016. DOI:10.1182/ blood-2016-07-726729.

10. May RE, Strong R. Acute emphysematous cholecystitis. Br J Surg 1971;58:453-458.

11. Hernandez-Rodriguez J, Tan CD, Rodriguez ER, et al. Singleorgan gallbladder vasulitis: Characterization and distinction from systemic vasculitis involving the gallbladder. An analysis of 61 patients. Medicine 2014;405-413.

Address correspondence to: Dr. Allison E. Berndtson

200 West Arbor Drive, Mail Code 8896

San Diego, CA 92103-8896

E-mail: aberndtson@ucsd.edu

$\begin{aligned} & \text { Abbreviations Used } \\ \mathrm{CT} & =\text { computed tomography } \\ \mathrm{HIV} & =\text { human immunodeficiency virus } \\ \mathrm{IV} & =\text { intravenous } \\ \mathrm{TPN} & =\text { total parenteral nutrition }\end{aligned}$

Cite this article as: Berndtson $\mathrm{AE}$, Hamel MG,

Costantini TW, Coimbra R (2017) Acalculous perforated hemorrhagic emphysematous cholecystitis caused by Clostridium perfringens in a patient with myeloma taking daratumumab. Surgical Infections Case Reports 2:1, 1-4, DOI: $10.1089 /$ crsi.2016.0050 